\title{
Capacity Scaling in Delay Tolerant Networks with Heterogeneous Mobile Nodes
}

\author{
Michele Garetto \\ Università di Torino,Italy \\ michele.garetto@unito.it
}

\author{
Paolo Giaccone \\ Politecnico di Torino,Italy \\ paolo.giaccone@polito.it
}

\author{
Emilio Leonardi \\ Politecnico di Torino,Italy \\ emilio.leonardi@polito.it
}

\begin{abstract}
We provide a general framework for the analysis of the capacity scaling properties in mobile ad-hoc networks with heterogeneous nodes and spatial inhomogeneities. Existing analytical studies strongly rely on the assumption that nodes are identical and uniformly visit the entire network space. Experimental data, however, have shown that the mobility pattern of individual nodes is typically restricted over the area, while the overall node density is often largely inhomogeneous, due to prevailing clustering behavior resulting from hot-spots. Such ubiquitous features of realistic mobility processes demand to reconsider the scaling laws for the peruser throughput achievable by the store-carry-forward communication paradigm which provides the foundation of many promising applications of delay tolerant networking. We show how the analysis of the asymptotic capacity of dense mobile ad-hoc networks can be transformed, under mild assumptions, into a Maximum Concurrent Flow (MCF) problem over an associated Generalized Random Geometric Graph (GRGG). Our methodology allows to identify the scaling laws for a general class of mobile wireless networks, and to precisely determine under which conditions the mobility of nodes can indeed be exploited to increase the per-node throughput. At last we propose a simple, asymptotically optimal, scheduling and routing scheme that achieves the maximum transport capacity of the network.
\end{abstract}

\section{Categories and Subject Descriptors}

C.2.1 [Computer-Communication Networks]: Network Architecture and Design-Wireless Communication

\section{General Terms}

Performance, Design, Theory

\section{Keywords}

Delay Tolerant Networking, Routing, Network Capacity

\section{INTRODUCTION}

Scalability has been the most fundamental concern that has so far discouraged the deployment of large-scale ad hoc networks. Among several scalability issues, perhaps the most basic one is that

Permission to make digital or hard copies of all or part of this work for personal or classroom use is granted without fee provided that copies are not made or distributed for profit or commercial advantage and that copies bear this notice and the full citation on the first page. To copy otherwise, to republish, to post on servers or to redistribute to lists, requires prior specific permission and/or a fee.

MobiHoc'07, September 9-14, 2007, Montréal, Québec, Canada.

Copyright 2007 ACM 978-1-59593-684-4/07/0009 ...\$5.00. related to the data transport capability of ad hoc networks comprising a large number of nodes establishing random connections among them. In their seminal work, Gupta and Kumar [1] obtained the disheartening result that, for a wireless network with $n$ static nodes, the per node throughput decays as $1 / \sqrt{n}$, even allowing optimal scheduling and node placement.

Later on, Grossglauser and Tse [2] have shown that a constant per node throughput can be achieved in the case of mobile nodes, by exploiting a novel store-carry-forward communication paradigm according to which data are physically carried on the nodes as they move around the network area. Although this communication scheme incurs very large delays, on the time scale of nodes movement across the network space, it has laid the foundation of an entire new area of research, usually referred to as delay-tolerant or disruption-tolerant networking (DTN) [3], which has recently attracted a lot of attention. A typical DTN scenario consists of a sparse set of fixed or mobile nodes, and it is characterized by intermittent connectivity and frequent network partitioning, such that node mobility is indeed essential to guarantee network cohesion and end-to-end communication.

Several interesting applications of DTN have been already envisioned and experimented upon, such as "pocket switched networks" based on human mobility [4], vehicular networks based on public buses [5] or taxi cabs [6], sensor networks for wildlife tracking [7], disaster-relief networks [8], Internet access to remote or rural villages [9]. While the theoretical result of Grossglauser and Tse suggests that delay tolerant networks can indeed scale up to very large sizes, it is unclear whether this result indeed applies to reallife scenarios such as the ones mentioned above. The main reason is that the analysis in [2] strongly relies on the assumptions that: i) all nodes are identical; ii) each node uniformly visits the entire network area according to an ergodic mobility process; iii) the trajectories of different nodes are independent and identically distributed (i.i.d.). Notice that the same hypotheses have been kept in the several papers that, after [2], have analyzed asymptotic delay-capacity trade-offs, like [10, 11, 12].

In many practical settings, the above assumptions on the nodes behavior do not hold, and in particular the one that the mobility process of each node uniformly covers the entire space over time, making all nodes basically indistinguishable from each other. Both everyday life experience and campus- or city-wide traces containing spatial information (i.e., based on GPS coordinates or radio beacons from base stations and access points) $[6,13,14,15]$, suggest that a node spends most of the time just in a small portion of the network area, comprising a few frequently visited "significant places" [16], and rarely goes outside this region. Although any two nodes are likely, in the long run, to eventually come in contact with each other, the impact of rare contacts on the overall network capacity has to be carefully investigated.

Moreover, not only nodes are characterized by rather restricted 
mobility processes, but also the overall node density over the area is largely inhomogeneous, and typically exhibits "concentration points" [6] or hotspots [17] where nodes are more likely to gather. Such clustering behavior has been observed in many different traces related to both human and vehicular movements, and appears to be a quite ubiquitous feature of real mobility processes. Examples of concentrations points include workplaces, restaurants, movie theaters, conference rooms (in the case of people), watering holes, oases (in the case of animals), intersections, parking lots, gas stations (in the case of road traffic).

The aforementioned characteristics of real life mobility processes demand to reconsider the study of the asymptotic capacity of mobile ad hoc networks originally proposed in [2], by explicitly accounting for the most common features recognized in real traces. Some progress has been already done in the direction of considering special cases of restricted mobility. In [18] the authors have considered a mobility pattern according to which each node independently moves along a randomly chosen great circle on the sphere. Quite surprisingly, even under this one-dimensional mobility pattern a constant throughput per source-destination pair can be sustained. In [19] the network of unit area is partitioned into square cells, and nodes are restricted to move within one randomly chosen cell, whose area is assumed to either scale as $(\log n) / n$ or remain constant. In [20] the authors study the capacity of an arbitrary network consisting of a finite number of mobile nodes. They prove that the capacity region is convex, it depends only on the stationary node distribution, and that maximum throughput can be achieved by the simple class of scheduling policies based just on position information. They also consider a few examples of anisotropic node stationary distributions and derive an upper bound for the resulting asymptotic network capacity. However, asymptotic results in [20] have been obtained exploiting ad hoc intuitive arguments on particular cases, moreover no routing scheme is proposed to approach the asymptotic network capacity.

In the special case of static nodes, several papers have already appeared which generalize the results of Gupta-Kumar to networks of heterogeneous nodes. The simple approach in [21] allows to consider nodes located over straight lines or highly dense neighborhoods. The impact of bottlenecks due to asymmetric traffic conditions or node clusters has been studied in [22]. In [23] the authors analyze the impact of directional antennas on the asymptotic network capacity using a technique similar to ours. Several works have also considered the capacity of hybrid networks comprising a mixture of wired and wireless nodes [24, 25, 26].

\subsection{Our Contribution}

The goal of this paper is to provide a general framework for the analysis of the capacity scaling properties in dense mobile ad-hoc networks with heterogenous nodes and spatial inhomogeneities, thus extending and generalizing the results reported in $[2,18,20]$. The contributions of this paper are:

- we show how the problem of establishing the asymptotic capacity of dense mobile ad-hoc networks can be mapped, under mild assumptions, into a Maximum Concurrent Flow (MCF) problem [28] over an associated Generalized Random Geometric Graph (GRGG).

- we develop a technique to solve the MCF problem over GRGs. Our methodology can be successfully applied to exactly evaluate the asymptotic capacity of a wide range of mobile wireless networks.

- we introduce a simple, asymptotically optimal, scheduling and routing scheme that achieves the maximum transport capacity of the network.

\section{SYSTEM ASSUMPTIONS AND NOTA- TION}

\subsection{Mobility Model}

We consider a network composed of $n$ nodes moving over a bidimensional Torus surface $\mathcal{O} .^{1}$

Let $X_{i}(t)$ denote the position of node $i$ at time $t$ and $X(t)=$ $\left(X_{1}(t), X_{2}(t) \ldots X_{n}(t)\right)$ be the vector of nodes' positions; we define by $d_{i j}(t)$ the distance between mobile $i$ and mobile $j$ at time $t$, i.e. ${ }^{2}: d_{i j}(t)=\left\|X_{i}(t)-X_{j}(t)\right\|$

Heterogeneity of nodes is taken into account at two different levels, based on common features that have been widely recognized in realistic mobility traces.

First, we consider that the stationary spatial distribution of a node is generally non-uniform over the space; rather, a node typically spends most of the time in a small region of the network area, and rarely (or never) visits zones far away from it. We model this behavior assuming that each node $i$ has a home-point, located at $X_{i}^{h}$, where its spatial stationary distribution is maximum. Nodes move "around" their home-point according to independent stationary and ergodic processes, i.e., given any $m$-uple $\left(B_{1}, B_{2}, B_{3} \ldots B_{m}\right)$ of Lebesgue measurable subsets of $\mathcal{O}$, it results (w.p.1):

$$
\lim _{t \rightarrow \infty} \frac{1}{t} \int_{0}^{t} \mathbb{I}_{\left(\cap_{i} X_{i}(\tau) \in B_{i}\right)} d \tau=\cap_{i} E\left[\mathbb{I}_{\left(X_{i}(t) \in B_{i}\right)} \mid \mathcal{F}_{n}\right]
$$

being $\mathbb{I}$ the logical indicator function and $\mathcal{F}_{n}$ the Borel-field generated by $\left\{X_{i}^{h}\right\}_{i=1}^{n}$.

We describe the density of presence of node $i$ around $X_{i}^{h}$ by a function $\phi_{i}(X)=\phi\left(X-X_{i}^{h}\right)$, which is assumed to be invariant in all directions. More specifically, we assume that the shape of $\phi_{i}(X)$ is given by a non-increasing, summable function $s(d)$ of the distance $d \in \mathbb{R}^{+}$from the home-point $X_{i}^{h}$. To obtain a proper probability density function over the area, we normalize $\phi_{i}(X)$ as follows,

$$
\phi_{i}(X)=\phi\left(X-X_{i}^{h}\right)=\frac{s\left(\left\|X-X_{i}^{h}\right\|\right)}{\int_{\mathcal{O}} s\left(\left\|X-X_{i}^{h}\right\|\right) d X}
$$

Note that, as limit case, we can obtain networks with static nodes; in this case $\phi_{i}(X)=\delta\left(X-X_{i}^{h}\right)$, being $\delta(X)$ the Dirac impulse function. For simplicity, we assume that the mobility of all nodes is characterized by the same function $\phi(X)$. However, this restriction can be relaxed introducing classes of nodes with different functions $\phi(X)$. Indeed our analysis can be easily extended to the case in which nodes with different mobility patterns coexist (e.g., a mixture of fixed nodes and fully mobile nodes, or several classes of nodes with different degrees of mobility around their home-points).

Second, we account for spatial inhomogeneities of the overall density of nodes over the area. To do so, we distribute the home points of the nodes according to two different models:

- Uniform: home-points of nodes are uniformly and independently chosen inside area $\mathcal{O}$.

- Clustered: the $n$ nodes are partitioned into $m$ clusters according to i.i.d. random variables. Each cluster has a middle point which is uniformly located within $\mathcal{O}$. The home-points

\footnotetext{
${ }^{1}$ The bidimensional (topological) Torus is the surface generated by the Cartesian product of two circles of unitary length. The Torus can be equivalently described as a quotient of the Cartesian plane under the identifications $(x, y) \simeq(x+1, y) \simeq(x, y+1)$.

${ }^{2}$ Given any two points $X_{1}=\left(x_{1}, y_{1}\right) \in \mathcal{O}$ and $X_{2}=$ $\left(x_{2}, y_{2}\right) \in \mathcal{O}$ we define their distance according to: $d\left(X_{1}, X_{2}\right)=$ $\min _{u, v \in\{-1,0,1\}} \sqrt{\left(x_{1}+u-x_{2}\right)^{2}+\left(y_{1}+v-y_{2}\right)^{2}}$
} 


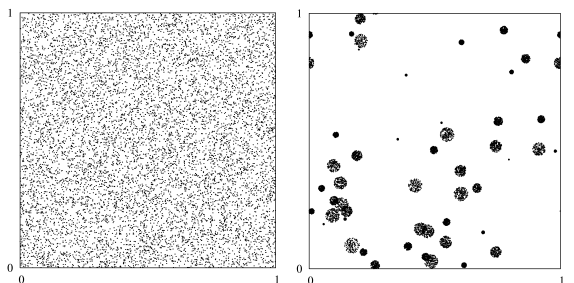

Figure 1: Examples of home-point distributions according to Uniform model (left plot) and Clustered model (right plot), in the case of $n=10,000$ nodes.

of nodes belonging to the same cluster are then uniformly and independently placed within a disk of radius $r$ centered at the cluster middle point.

The Uniform model, which is simpler to analyze, has been widely used in the literature to study random networks of static nodes. However, it does not take into account the clustering behavior that has been observed in real traces $[6,17]$. The Clustered model, instead, better captures the fact that in realistic scenarios the distribution of users on the territory may be highly inhomogeneous: parts of the network area are more densely populated than others, creating concentration points that are usually well distinct from each other and fairly stable over time. Examples of homepoint distributions according to Uniform and Clustered models are shown in Fig. 1 for $n=10,000$.

\subsection{Interference Model}

We assume that interference among simultaneous transmissions is described by the well known protocol model [1], which roughly represents the behavior of wireless MAC protocols in the case of omni-directional antennas without power capture. Nodes employ a common range $R_{T}$ for all their transmissions (equivalently, they employ a common power level, i.e., no power adaptation mechanism is used). Node $i$ is allowed to transmit to node $j$ at time $t$, only if: i) the distance between $i$ and $j$ is no more than $R_{T}$, i.e., $d_{i j}(t)<R_{T}$; ii) for every other node $k$ simultaneously transmitting, $d_{k j}(t)>(1+\Delta) R_{T}$, being $\Delta$ a guard factor. We assume that transmissions occur at fixed rate which is normalized to 1 .

\subsection{Traffic Model}

Similarly to previous work we consider uniform permutation traffic matrices, i.e., traffic patterns in which $n$ randomly selected source-destination pairs $(s, d)$ exchange traffic at rate $\lambda$. Sourcedestination pairs are selected is such a way that every node is origin and destination of a single traffic flow with average rate $\lambda$.

\section{ASYMPTOTIC ANALYSIS}

To analyze asymptotic properties as the network grows large, we progressively increase the number of nodes $n$, generating a sequence of systems indexed by $n$. We are essentially interested in establishing how the network capacity scales with $n$ under the assumptions we have introduced on node mobility, interference and traffic. We say that the per-node capacity (or maximum per-node throughput) of the system is ${ }^{3} \Theta(h(n))$ if, given a sequence of uniform permutation traffic patterns with rate $\lambda^{(n)}=h(n)$, there exist

${ }^{3}$ Given two functions $f(n) \geq 0$ and $g(n) \geq 0: \quad f(n)=$ $o(g(n))$ means $\lim _{n \rightarrow \infty} f(n) / g(n)=0 ; f(n)=O(g(n))$ means $\limsup _{n \rightarrow \infty} f(n) / g(n)=c<\infty ; f(n)=\omega(g(n))$ is equivalent to $g(n)=o(f(n)) ; f(n)=\Omega(g(n))$ is equivalent to $g(n)=O(f(n)) ; f(n)=\Theta(g(n))$ means $f(n)=O(g(n))$ and $g(n)=O(f(n))$; at last $f(n) \sim g(n)$ means $\lim _{n \rightarrow \infty} \frac{f(n)}{g(n)}=1$ two constants $c, c^{\prime}$ such that $c<c^{\prime}$ and both the following properties hold:

$$
\left\{\begin{array}{l}
\lim _{n \rightarrow \infty} \operatorname{Pr}\left\{c \lambda^{(n)} \text { is sustainable }\right\}=1 \\
\lim _{n \rightarrow \infty} \operatorname{Pr}\left\{c^{\prime} \lambda^{(n)} \text { is sustainable }\right\}<1
\end{array}\right.
$$

Equivalently, we say in this case that the network capacity (or maximum network throughput) is $\Theta(n h(n))$.

\subsection{Scaling the Network Size}

While the mobility pattern of nodes can well be considered to be independent of $n$ (i.e., the mobility process of users is exogenous to the system), the physical extension of a network depends on $n$, and typically increases as $n$ increases. This is actually the key point that determines how the network capacity scales with $n$, as we will see. Let $f(n)$ be a non decreasing function which characterizes how the physical extension of the network scales with $n$. Throughout the paper we will assume that $f(n)$ has the form $f(n)=n^{\alpha}$ with $\alpha \in[0,1 / 2]{ }^{4}$

When $\alpha=0$ we obtain the special case in which the network physical extension remains constant, while the node density increases linearly with $n$. In this scenario a node gets in contact with $\Theta(n)$ other nodes (a constant fraction of the entire population of users). We will see that, in this case, the network asymptotic capacity scales as if the nodes mobility process were uniform over the area $\mathcal{O}$ (Grossglauser-Tse case).

When $\alpha=1 / 2$, or, more in general, $f(n)=\Theta(\sqrt{n})$, we obtain the other extreme case in which the area size increases linearly with $n$, while the node density is kept independent on $n$. In this scenario a node gets in contact with $\Theta(1)$ other nodes (a finite number of nodes). We will see that, in this case, the asymptotic network capacity scales as if nodes were static (Gupta-Kumar case).

In this paper we are concerned with all intermediate cases as well. Since we normalize the network area to 1 , we need to properly scale down the spatial stationary distribution of the users (1), as the number of nodes increases. In particular, we need to progressively reduce the area spanned by each node, in accordance to how the physical extension of the network scales up with $n$. We obtain the sequence of spatial distributions

$$
\phi_{i}^{n}(X)=\phi^{n}\left(X-X_{i}^{h}\right)=\frac{s\left(f(n)\left\|X-X_{i}^{h}\right\|\right)}{\int_{\mathcal{O}} s\left(f(n)\left\|X-X_{i}^{h}\right\|\right) d X}
$$

Since

$$
\int_{\mathcal{O}} s\left(f(n)\left\|X-X_{i}^{h}\right\|\right) d X \sim 1 / f^{2}(n)
$$

it results:

$$
\phi_{i}^{n}(X) \sim f^{2}(n) s\left(f(n)\left\|X-X_{i}^{h}\right\|\right)
$$

When considering the Clustered model we also need to specify how parameters $m$ and $r$ scale with $n$. In this paper we will focus on clustered models in which $m(n)=\Theta\left(n^{\nu}\right)$ with $\nu \in[0,1)$ and $r(n)=\Theta\left(n^{-\beta}\right)$, with $\beta \in[0, \infty)$, under the constraint that $\nu-2 \beta<0$. In this case the different clusters are, with high probability, non-overlapping. Note that the Uniform model can be regarded as a limit case of the Clustered model where the number of clusters is $n$ (i.e., $\nu=1$ ), and, in addition, every cluster contains deterministically a single node.

\subsection{Scaling the Transmission Range}

A final consideration regards how the transmission range $R_{T}$ scales with $n$. Previous investigations $[1,2,20]$ have shown that transmission ranges should be reduced as much as possible so as

\footnotetext{
${ }^{4}$ We do not consider cases in which $\alpha>1 / 2$, which, however, can be proved to be equivalent to the case $\alpha=1 / 2$ in terms of capacity scaling properties.
} 
to increase the overall network capacity (and, at the same time, reduce the power consumption of nodes), while maintaining network connectivity.

In this paper we will generalize previous findings showing that, in a wide range of contexts, choosing $R_{T}=\Theta\left(\frac{1}{\sqrt{n}}\right)$ indeed maximizes the overall network capacity.

\section{MAIN RESULTS}

Consider a sequence of networks with increasing number of nodes $n$ in which nodes' home-points are placed either according to the Uniform model or to a $(m, r)$ Clustered model. We define $\gamma(n)=\frac{\log (m(n))}{m(n)}(m(n)=n$ in the Uniform model). Quantity $\sqrt{\gamma(n)}$ represents the minimal transmission range that would guarantee network connectivity in the case nodes remained still at their home-points. Two different asymptotic regimes appear depending on how $f(n)$ scales with respect to $\sqrt{\gamma(n)}$, as $n \rightarrow \infty$.

\subsection{Super-critical Regime}

This regime occurs when $f(n) \sqrt{\gamma(n)}=o(1)$. Here, mobility plays a fundamental role in favoring the exchange of information between nodes. Indeed node pairs whose home-points are separated by a distance $O\left(\frac{1}{f(n)}\right)$, because of mobility can get in contact with each other and thus have the opportunity of directly exchanging data.

The main properties of this regime are:

- The per-node capacity is $\Theta\left(\frac{1}{f(n)}\right)$ with high probability ${ }^{5}$ (w.h.p.), independently of both the shape $s(d)$ of the nodes' spatial distribution and of the parameters $m(n), r(n)$ of the particular Clustered model.

- The maximum network capacity is achieved by an implementable scheduling/routing scheme (specified later) in which the transmission range is $\Theta\left(\frac{1}{\sqrt{n}}\right)$.

- The implementation of the proposed optimal scheduling/routing scheme does not require the nodes to know the shape of $s(d)$, neither the parameters $m(n)$ and $r(n)$ associated to the distribution of home-points. They only need to know how $f(n)$ scales with $n$.

\subsection{Sub-critical Regime}

This regime occurs when $f(n) \sqrt{\gamma(n)}=\omega(1)$. Here, the impact of mobility on system performance is much less important, and a transmission range $R_{T}(n)=\Theta(\sqrt{\gamma(n)})$ may be required to guarantee network connectivity. In general, the network capacity becomes sensitive to both the shape of $s(d)$ and to the parameters $m(n), r(n)$ of the specific Clustered model. In this paper, being mainly interested in studying the asymptotic properties of the super-critical phase, we have limited our investigation on the sub-critical regime. We provide the following lower bound to the per-node capacity in sub-critical regime,

$$
\Theta\left(\frac{1}{n \sqrt{\gamma(n)}}\right)=\Theta\left(\sqrt{\frac{m(n)}{n^{2} \log (m(n))}}\right)
$$

showing that this lower bound is tight at least for one significant class of spatial distributions $\phi(X)$, i.e., the ones associated to functions $s(d)$ having finite support. We leave for future work a more in-depth analysis of the sub-critical capacity resulting from arbitrary spatial distributions $\phi(X)$.

$\overline{{ }^{5} \text { I.e., with a probability tending to } 1 \text { as } n \rightarrow \infty}$

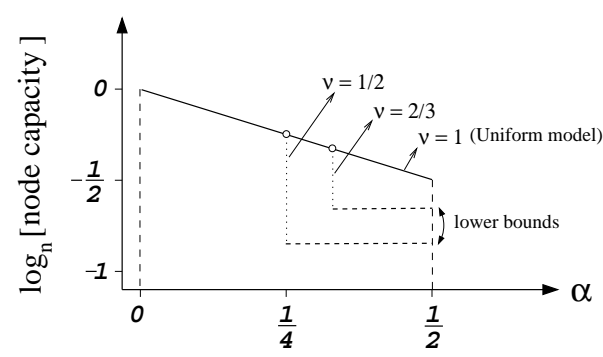

Figure 2: Per-node asymptotic capacity as a function of $f(n)=$ $n^{\alpha}$ for Uniform and Clustered models. Lower bounds are tight when function $s(d)$ has finite support.

\subsection{Graphical Representation of Capacity Results}

A graphical representation of our findings on asymptotic network capacity is reported in Fig. 2, where we show the per-node capacity as a function of $f(n)=n^{\alpha}$, for different values of the parameter $\nu$ of the Clustered model (recall that the number of clusters is $m=\Theta\left(n^{\nu}\right)$ ).

While in super-critical regime the per-node capacity smoothly increases while reducing $\alpha$, independently from the parameters of the clustered model and the shape of $s(d)$, in sub-critical regime the behavior is more complex; the per-node capacity becomes sensitive to both the parameters of the clustered model and the shape of $s(d)$. In the worst case (i.e., when $s(d)$ has finite support) the per-node capacity in the sub-critical region does not depend on $\alpha$, and a sharp phase transition occurs between the two regimes (represented in the figure by dotted vertical lines).

\section{ANALYSIS IN SUPER-CRITICAL REGIME}

Given any point $X_{0} \in \mathcal{O}$, we define the local density of nodes in $X_{0}$ as:

$$
\rho^{n}\left(X_{0}\right)=\sum_{i=1}^{n} E\left[\mathbb{I}_{X_{i}^{(n)}(t) \in B\left(X_{0}, 1 / \sqrt{n}\right)} \mid \mathcal{F}_{n}\right]
$$

where $B\left(X_{0}, 1 / \sqrt{n}\right)$ is the disk centered in $X_{0}$, of radius $1 / \sqrt{n}$. The asymptotic local density in $X_{0}$ is $\rho\left(X_{0}\right)=\lim _{n \rightarrow \infty} \rho^{n}\left(X_{0}\right)$. We emphasize that the asymptotic local density of nodes in $X_{0}$ is a non trivial random variable over the Borel-field generated by the nodes home-points locations $\left\{X_{i}^{h}\right\}$. Note that, averaging over all possible home point locations, we obtain $E_{\mathcal{F}_{n}}\left[\rho^{n}\left(X_{0}\right)\right]=\pi$ independently of $X_{0}$, thanks to the spatial homogeneity (on average) of both the Uniform and Clustered model. Theorem 1 provides a characterization of the asymptotic local density in super-critical regime:

Theorem 1. If $f(n) \sqrt{\gamma(n)}=o(1)$, then for any $X_{0} \in \mathcal{O}$ it is possible to find two positive constants $m, M$ such that $m \leq \pi \leq$ $M$ and

$$
m<\rho^{n}\left(X_{0}\right)<M \quad \text { w.h.p. }
$$

To prove the theorem we need to premise a useful result whose proof is reported in Appendix A.

Lemma 1. Suppose that $\left\{X_{i}^{h}\right\}_{i=1}^{n}$ are displaced on $\mathcal{O}$ either according to the Uniform model or according to a $(m(n), r(n))$ Clustered model such that $\lim _{n \rightarrow \infty} m(n) r^{2}(n)=0$. Given a sequence of regular tessellations $\mathcal{A}^{n}$ of $\mathcal{O}$ (or any its sub-region), with the property that the area of each element of the tessellation $\left|A^{n}\right| \geq(16+\delta) \gamma(n)$, for some small $\delta>0$, and defined with 
$N\left(A_{k}^{n}\right)$ the number of home-points inside $A_{k}^{n}$, then uniformly over the tessellations w.h.p. $N\left(A_{k}^{n}\right)$ is comprised between $\frac{n\left|A^{n}\right|}{2}$ and $2 n\left|A^{n}\right|$, i.e., $\left.\frac{n\left|A^{n}\right|}{2}<\inf _{k} N\left(A_{k}^{n}\right) \leq \sup _{k} N\left(A_{k}^{n}\right)<2 n\left|A^{n}\right|\right)$.

Proof. (Theorem 1). Consider a generic point $X_{0} \in \mathcal{O}$. First suppose $s(d)$ to be continuous at every point.

$$
\begin{aligned}
& \rho^{n}\left(X_{0}\right)=f^{2}(n) \sum_{i=1}^{n} \int_{B\left(X_{0}, 1 / \sqrt{n}\right)} s\left(f(n)\left\|X-X_{i}^{h}\right\|\right) d X \sim \\
& \sim f^{2}(n)\left|B\left(X_{0}, 1 / \sqrt{n}\right)\right| \sum_{i=1}^{n} s\left(f(n)\left\|X_{0}-X_{i}^{h}\right\|\right)= \\
&=\frac{\pi f^{2}(n)}{n} \sum_{i=1}^{n} s\left(f(n)\left\|X_{0}-X_{i}^{h}\right\|\right)
\end{aligned}
$$

where the first equality holds for the continuity of function $s(d)$, being $\left|B\left(X_{0}, 1 / \sqrt{n}\right)\right|=\frac{\pi}{n}=o\left(\frac{1}{f^{2}(n)}\right)$. When $s(d)$ admits points at which it is not continuous, asymptotically for $n \rightarrow \infty$ it holds:

$$
\begin{array}{r}
\frac{\pi f^{2}(n)}{n} \sum_{i=1}^{n} s\left(f(n)\left\|X_{0}-X_{i}^{h}\right\|+\frac{f(n)}{n}\right)<\rho_{0}^{n}\left(X_{0}\right)< \\
\frac{\pi f^{2}(n)}{n} \sum_{i=1}^{n} s\left(f(n)\left\|X_{0}-X_{i}^{h}\right\|-\frac{f(n)}{n}\right)
\end{array}
$$

Now, let $\mathcal{A}^{n}$ denote a regular square tessellation of $\mathcal{O}$, such that each square $A_{k}^{n}$ of $\mathcal{A}^{n}$ has area $\left|A^{n}\right|=(16+\delta) \gamma(n)$. Let $\underline{d}_{0 k}$ and $\bar{d}_{0 k}$ be, respectively, the inferior and the superior of the distances between points $X \in A_{k}$ and $X_{0}$; i.e., $\underline{d}_{0 k}=\inf _{X \in A_{k}^{n}}\left\|X-X_{0}\right\|$; and $\bar{d}_{0 k}=\sup _{X \in A_{k}^{n}}\left\|X-X_{0}\right\|$; at last, let $\underline{N}\left(A_{k}^{n}\right)$ and $\bar{N}\left(A_{k}^{n}\right)$ be, respectively, a lower bound and an upper bound of the number of mobiles whose home-point falls within $A_{k}^{n}$. It results:

$$
\begin{array}{r}
\frac{\pi f^{2}(n)}{n} \sum_{k} s\left(f(n) \bar{d}_{0 k}\right) \underline{N}\left(A_{k}^{n}\right)< \\
<\rho_{0}^{n}\left(X_{0}\right)<\frac{\pi f^{2}(n)}{n} \sum_{k} s\left(f(n) \underline{d}_{0 k}\right) \bar{N}\left(A_{k}^{n}\right)
\end{array}
$$

Moreover since w.h.p., uniformly over $k, \underline{N}\left(A_{k}^{n}\right) \geq n / 2\left|A^{n}\right|$ and $\bar{N}\left(A_{k}\right) \leq 2 n\left|A^{n}\right|$ (Lemma 1), we get the assert.

Indeed, observe that, i) $f^{2}(n) \sum_{k} s\left(f(n) \bar{d}_{0 k}\right)\left|A^{n}\right|$ and $f^{2}(n) \sum_{k} s\left(f(n) \underline{d}_{0 k}\right)\left|A^{n}\right|$ can be interpreted, respectively, as lower Riemann sum and upper Riemann sum of $\int_{\mathcal{O}} s(f(n) \| X-$ $\left.X_{0} \|\right) d X$; ii) since $f(n) \sqrt{\gamma(n)}=o(1)$, the mesh size of the partitions associated to Riemann sums vanishes to 0 as $n \rightarrow \infty$. As a result:

$\pi f^{2}(n) \sum_{k} s\left(f(n) \bar{d}_{0 k}\right)\left|A^{n}\right| \sim \pi f^{2}(n) \sum_{k} s\left(f(n) \underline{d}_{0 k}\right)\left|A^{n}\right| \sim$

\subsection{Scheduling Policy}

$$
\sim \pi \int_{\mathbb{R}^{2}} s\left(\left\|X-X_{0}\right\|\right) d X=\pi
$$

The scheduling policy $S$ is in charge to dynamically select at each time an implementable transmission configuration, i.e., to select a set $\pi^{S}(t)$ of non-interfering node pairs, which are allowed to simultaneously communicate. In this paper we restrict our investigation to stationary and ergodic scheduling policies, i.e, policies for which the capacity $\mu_{i j}^{S}$ between any two nodes $i$ and $j$ satisfies (w.p.1):

$$
\mu_{i j}^{S}=E\left[\mathbb{I}_{(i, j) \in \pi^{S}(t)} \mid \mathcal{F}_{n}\right]=\lim _{t \rightarrow \infty} \frac{1}{t} \int_{0}^{t} \mathbb{I}_{(i, j) \in \pi^{S}(\tau)} d \tau
$$

In general, the selection of $\pi^{S}(t)$ may be influenced by several parameters, including instantaneous queues lengths, age of stored information at nodes, etc. In [20] it has been proven that the class of position-based scheduling policies, i.e., those policies in which the selection of the transmission configuration $\pi^{S}(t)$ is driven only by the vector of instantaneous nodes' positions $X(t)$, achieves the maximum network capacity.

In particular, in this work we consider the following positionbased scheduling policy, which complies with the conditions imposed by the protocol interference model:

Definition 1. Given a network comprising $n$ nodes, policy $S^{*}$ enables transmission between node $i$ and node $j$ when the following conditions are satisfied:

$$
\begin{aligned}
d_{i j}(t) & <R_{T}=\frac{c_{T}}{\sqrt{n}} \\
\min \left(d_{k j}(t), d_{k i}(t)\right) & >(1+\Delta) R_{T}
\end{aligned}
$$

for every other node $k$ in the network (regardless of node $k$ activity), where $c_{T}$ is a constant. Moreover, the transmission bandwidth between $i$ and $j$ is equally shared in the two directions.

Long term capacities achieved by $S^{*}$ in a network comprising $n$ nodes will be denoted by $\mu_{i j}^{* n}$. They can be expressed as function of the stationary spatial distribution of nodes as follows:

$$
\begin{array}{r}
\mu_{i j}^{* n}=\frac{1}{2} \int_{X_{i} \in \mathcal{O}} \int_{X_{j} \in B\left(X_{i}, c_{T} / \sqrt{n}\right)}\left[\prod_{k \neq i, j} \int_{X_{k} \in A_{\Delta}\left(X_{i}, X_{j}\right)} \phi_{k}\left(X_{k}\right)\right. \\
\left.d X_{k}\right] \phi_{j}\left(X_{j}\right) d X_{j} \phi_{i}\left(X_{i}\right) d X_{i}
\end{array}
$$

being $A_{\Delta}\left(X_{i}, X_{j}\right)$ the region defined by the set of points $\left\{X: \min \left(\left\|X-X_{j}\right\|,\left\|X-X_{i}\right\|\right)>(1+\Delta) \frac{c_{T}}{\sqrt{n}}\right\}$.

Theorem 2. Under policy $S^{*}$, if (3) holds, for any pair of nodes $(i, j)$ and any finite $c_{T}>0$ :

$$
\mu_{i j}^{* n}=\Theta\left(\operatorname{Pr}\left\{d_{i j} \leq \frac{c_{T}}{\sqrt{n}} \mid \mathcal{F}_{i j}\right\}\right)
$$

where $\mathcal{F}_{i j}$ is the Borel-field generated by the variables $X_{i}^{h}$ and $X_{j}^{h}$. The proof is reported in Appendix B.

Theorem 2 allows us to say that, in the super-critical regime, if policy $S^{*}$ is adopted, the long-term capacity between two nodes is of the same order as the fraction of time during which one node falls within the transmission range of the other. As a consequence: i) no other scheduling policy employing a transmission range $R_{T}(n)=\Theta\left(\frac{1}{\sqrt{n}}\right)$ can achieve an asymptotically higher throughput (in order sense) than $S^{*}$; ii) to evaluate $\mu_{i j}^{* n}$ (in order sense), we can reduce ourselves to computing the "contact probabilities' $\operatorname{Pr}\left\{d_{i j} \leq R_{T}(n)\right\}$ :

$$
\begin{gathered}
\operatorname{Pr}\left\{d_{i j} \leq R_{T}(n)\right\} \sim \\
f^{4}(n) \int_{X_{i} \in \mathcal{O}} \int_{X_{j} \in B\left(X_{i}, R_{T}(n)\right)} s\left(f(n)\left\|X_{j}-X_{j}^{h}\right\|\right) \\
s\left(f(n)\left\|X_{i}-X_{i}^{h}\right\|\right) d X_{i} d X_{j} \sim \\
f^{4}(n)\left|B\left(0, R_{T}(n)\right)\right| \int_{X \in \mathcal{O}} s\left(f(n)\left\|X-\left(X_{j}^{h}-X_{i}^{h}\right)\right\|\right) \\
s(f(n)\|X\|) d X= \\
\pi R_{T}^{2}(n) f^{4}(n) \int_{X \in \mathbb{R}^{2}} s\left(f(n)\left\|X-\left(X_{j}^{h}-X_{i}^{h}\right)\right\|\right) s(f(n)\|X\|) d X \\
=\pi R_{T}^{2}(n) f^{2}(n) \int_{Y \in \mathbb{R}^{2}} s\left(\left\|Y-f(n)\left(X_{j}^{h}-X_{i}^{h}\right)\right\|\right) s(\|Y\|) d Y
\end{gathered}
$$


where the first equality holds thanks to the almost everywhere continuity of function $s(d)$, and the second because $s(d)$ is summable. Being $R_{T}(n)=\frac{c_{T}}{\sqrt{n}}$, thanks to Theorem 2 it results,

$$
\mu_{i j}^{n}=\Theta\left(g(n) \eta\left(f(n)\left\|X_{j}^{h}-X_{i}^{h}\right\|\right)\right)
$$

having defined:

$$
\eta(\|Y\|)=\int_{X \in \mathbb{R}^{2}} s(\|X-Y\|) s(\|X\|) d X
$$

and $g(n)=\pi c_{T}^{2} \frac{f^{2}(n)}{n}$.

With abuse of notation, we define also $\eta(d)$, with $d \in \mathbb{R}^{+}$, as $\eta(\|Y\|)$ for any $Y$ such that $\|Y\|=d$. Note that $\eta$ is a non-increasing, summable function $\mathbb{R}^{+} \rightarrow \mathbb{R}^{+}$which is everywhere continuous and derivable (with continuous derivative) at every point with the exception of at most a denumerable set of points.

\subsection{Mapping over Generalized Random Geometric Graph}

The asymptotic capacity achievable by a mobile wireless network under the constraints: i) $\sqrt{\gamma(n)} f(n)=o(1)$; ii) scheduling policy selects transmission ranges $O(1 / \sqrt{n})$, can be studied by representing the network as a Generalized Random Geometric Graph $G(n, m, r, \mu)$ defined as follows ${ }^{6}$ : the $n$ vertices of the graph stand for the home-points of the nodes, which are randomly located according to an $(m, r)$ Clustered model, (conventionally if $m=n$ we assume the home-points to be located according to the Uniform model); pair of vertices $(i, j)$ are connected by an edge (link) of capacity $\mu_{i j}^{n}$ which depends only on the distance between the corresponding home-points. In particular, we have $\mu_{i j}^{n}\left(d_{i j}\right)=g(n) \eta\left(f(n) d_{i j}\right)$ with $d_{i j}=\left\|X_{i}^{h}-X_{j}^{h}\right\|$ and $g(n)=\pi c_{T}^{2} \frac{f^{2}(n)}{n}$.

The network capacity under a traffic matrix $\boldsymbol{\Lambda}=\left[\lambda_{s d}\right]$, can be found by solving a Maximum Concurrent Flow (MCF) problem [28] over the associated GRGG, i.e. by solving the following multi-commodity flow problem:

$$
\left\{\begin{array}{l}
\max \lambda \\
\lambda \sum_{s} \sum_{d} \lambda_{s d} f_{i j}^{s d} \leq \mu_{i j}^{n}
\end{array}\right.
$$

with $f_{i j}^{s d} \in[0,1]$ denoting the average fraction of traffic from node $s$ to node $d$, which is routed through link $(i, j)$, i.e. $j$ follows $i$ as relay node along the path. We have the following routing continuity constraints:

$$
\sum_{i} f_{i j}^{s d}-\sum_{k} f_{j k}^{s d}=\left\{\begin{array}{cc}
1 & \text { for } j=d \\
0 & \text { for } j \neq d \text { and } j \neq s \\
-1 & \text { for } j=s
\end{array}\right.
$$

Note that $\lambda$ represents the maximum amount of traffic the network/graph can transport; the set $\left\{f_{i j}^{s d}\right\}$ univocally defines the corresponding routing strategy in the network/graph.

In general, MCF problems are hard to solve; an upper bound to $\lambda$ can be obtained in terms of graph cuts:

Proposition 1. Traffic $\lambda \boldsymbol{\Lambda}$ is sustainable only if, for any partition $(\mathcal{S}, \mathcal{D})$ of the nodes, it results:

$$
\lambda \sum_{s \in \mathcal{S}} \sum_{d \in \mathcal{D}} \lambda_{s d} \leq \sum_{i \in \mathcal{S}} \sum_{j \in \mathcal{D}} \mu_{i j}^{n}
$$

It was proven in [28] that, in undirected graphs, traffic is guaranteed to be sustainable if the ratio between the minimum value of the

${ }^{6}$ The class of GRGG generalizes the class of Random Geometric Graphs [27] r.h.s. and the maximum value of the 1.h.s. in (9) is $\Omega(\log k)$, being $k$ the number of flows.

In the case of GRGG node partitions can be obtained by physically partitioning the area $\mathcal{O}$ into two disjoint regions $\mathcal{S}^{\prime}$ and $\mathcal{D}^{\prime}$ :

$$
\lambda \leq \frac{\sum_{i: X_{j}^{h} \in \mathcal{S}^{\prime}} \sum_{j: X_{j}^{h} \in \mathcal{D}^{\prime}} \mu_{i j}^{n}}{\sum_{s: X_{s}^{h} \in \mathcal{S}^{\prime}} \sum_{d: X_{d}^{h} \in \mathcal{D}^{\prime}} \lambda_{s d}}
$$

When $\Lambda$ is a uniform permutation traffic matrix (without lack of generality we assume $\lambda_{s d} \in\{0,1\}$ ) the denominator of (10) counts the number of traffic relations with source $s \in \mathcal{S}^{\prime}$ and destination $d \in \mathcal{D}^{\prime}$.

\subsection{Effect of Transmission Range}

Looking at (6), it can be noticed that, by increasing the transmission range, one can increase the contact probability between any two nodes. However the potential advantage deriving from augmenting the transmission range is totally offset by the increased interference produced over possible concurrent transmissions. This fact can be easily shown in the case $\eta(d)$ (or, equivalently, $s(d)$ ) has finite support.

Theorem 3. In super-critical regime, the network capacity cannot be increased by increasing the transmission range beyond $\Theta\left(\frac{1}{\sqrt{n}}\right)$, if there exists an $y_{0}$ such that $\eta\left(y_{0}\right)=0$.

PROOF. Since there exists a finite $y_{0}$ such that $\eta(y)=0$ for $y \geq y_{0}, \operatorname{Pr}\left\{d_{i j} \leq R_{T}(n)\right\}>0$ only if $\left\|X_{j}^{h}-X_{i}^{h}\right\| \leq \frac{y_{0}}{f(n)}$. The radio interface of a node can, at most, be fully utilized all the time. Thus we have $\mu_{i}^{n}=\sum_{j} \mu_{i j}^{n} \leq 1$. Consider a line $\mathcal{L}$ dividing $\mathcal{O}$ in two regions $A_{0}$ and $B_{0}$. The capacity flow through the corresponding cut is $\sum_{i \in A_{0}} \sum_{j \in B_{0}} \mu_{i j}^{n}$. However, by construction, no edges exist between node $i \in A_{0}$ and any $j \in B_{0}$ if the distance between $X_{i}^{h}$ and line $\mathcal{L}$ is greater than $\frac{y_{0}}{f(n)}$. Thus defining with $C_{0}$ the region of points belonging to $A_{0}$ whose distance from $\mathcal{L}$ is less than $\frac{y_{0}}{f(n)}$.

$$
\begin{aligned}
\sum_{i \in A_{0}} \sum_{j \in B_{0}} \mu_{i j}^{n}=\sum_{i \in C_{0}} \sum_{j \in B_{0}} \mu_{i j}^{n} \leq \sum_{i \in C_{0}} \mu_{i}^{n} \leq \\
\leq N\left(C_{0}\right) \leq 2 n\left|C_{0}\right|=\Theta\left(\frac{n}{f(n)}\right)
\end{aligned}
$$

because of Lemma 1 and $\left|C_{0}\right|=\Theta\left(\frac{1}{f(n)}\right) \gg \sqrt{\gamma(n)}>\gamma(n)$. We conclude that $\lambda=O(1 / f(n))$. Comparing this capacity with that provided by policy $S^{*}$ (obtained in the next section) the assert follows.

Furthermore, policies adopting a transmission range $R_{T}=$ $\omega\left(\frac{1}{\sqrt{n}}\right)$ achieve a throughput which is strictly $o\left(\frac{1}{f(n)}\right)$; this is due to the fact that, when a node transmits, it prevents from transmitting all of the other nodes which are within its transmission range. The number of nodes within a transmission range scales as $\Theta\left(n R_{T}^{2}(n)\right)$ being the asymptotic local density finite and non-null at every point $X_{0}$ (Theorem 1). Thus since the number of transmission opportunities given to a node is inversely proportional to the number of nodes which are interfering with it, it results: $\frac{1}{n} \sum_{i=1}^{n} \mu_{i}^{n}=\Theta\left(\frac{1}{n R_{T}^{2}(n)}\right)$. This is the key observation that allows to extend Theorem 3 to the more general case in which $\eta(y)>0$ for every $y \in \mathbb{R}^{+}$. However, due to space constraints, we omit the complete proof for the general case.

\subsection{Computation of the Capacity of GRGG}



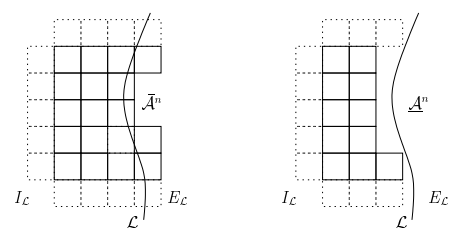

Figure 3: Example of outer (left) and inner (right) tessellations of $I_{\mathcal{L}}$. Similar tessellations can be drawn for $E_{\mathcal{L}}$

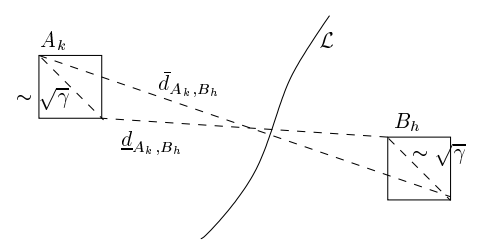

Figure 4: Definitions of the distances between $A_{k}$ and $B_{h}$

Given a random graph $G(n, m, r, \mu)$ and any simple, regular, closed curve $\mathcal{L}$ dividing $\mathcal{O}$ in two regions $I_{\mathcal{L}}$ and $E_{\mathcal{L}}$, we define the capacity crossing $\mathcal{L}$ as:

$$
\mu_{\mathcal{L}}^{n}=\sum_{i \in I_{\mathcal{L}}} \sum_{j \in E_{\mathcal{L}}} \mu^{n}\left(d_{i j}\right)
$$

Note that $\mu_{\mathcal{L}}^{n}$ is a random variable defined over $\mathcal{F}_{n}$, i.e., it depends on the locations of nodes' home-points $\left\{X_{i}^{h}\right\}_{i=1}^{n}$.

Averaging over all possible $\left\{X_{i}^{h}\right\}_{i=1}^{n}$, by spatial uniformity (on average) of Uniform and Clustered models, it immediately follows that:

$$
E\left[\mu_{\mathcal{L}}^{n}\right]=\frac{n^{2}}{\left|I_{\mathcal{L}} \| E_{\mathcal{L}}\right|} \int_{I_{\mathcal{L}}} \int_{E_{\mathcal{L}}} \mu^{n}(\|X-Y\|) d X d Y
$$

The fundamental point that we need to establish is whether:

$$
\mu_{\mathcal{L}}^{n}=\Theta\left(E\left[\mu_{\mathcal{L}}^{n}\right]\right) \quad \text { w.h.p. }
$$

By defining: $\overline{\mathcal{A}}^{n}$ and $\overline{\mathcal{B}}^{n}$ as square outer tessellations of $I_{\mathcal{L}}$ and $E_{\mathcal{L}}$, respectively (see Fig. 3); $\bar{N}\left(A_{k}\right)$ and $\bar{N}\left(B_{h}\right)$ as upper bounds on the number of vertices within $A_{k} \in \overline{\mathcal{A}}^{n}$ and $B_{h} \in \overline{\mathcal{B}}^{n} ; \underline{d}_{A_{k}, B_{h}}$ as the minimum distance between points of $A_{k}$ and $B_{h}$. Then, $\mu_{\mathcal{L}}^{n}$ can be upper bounded by:

$$
\sum_{A_{k} \in \overline{\mathcal{A}}^{n}} \sum_{B_{h} \in \overline{\mathcal{B}}^{n}} \mu^{n}\left(\underline{d}_{A_{k}, B_{h}}\right) \bar{N}\left(A_{k}\right) \bar{N}\left(B_{h}\right)
$$

Analogously, by defining: $\underline{A}^{n}$ and $\underline{\mathcal{B}}^{n}$ as square inner tessellations of $I_{\mathcal{L}}$ and $E_{\mathcal{L}}$, respectively (see again fig 3 ); $\underline{N}\left(A_{k}\right)$ and $\underline{N}\left(B_{h}\right)$ as lower bounds on the number of vertices within $A_{k} \in \overline{\mathcal{A}}^{n}$ and $B_{h} \in \underline{\mathcal{B}}^{n} ; \bar{d}_{A_{k}, B_{h}}$ as the maximum distance between points of $A_{k}$ and $B_{h}$. Then, $\mu_{\mathcal{L}}^{n}$ can be lower bounded by:

$$
\sum_{A_{k} \in \underline{A}^{n}} \sum_{B_{h} \in \underline{\mathcal{B}}^{n}} \mu^{n}\left(\bar{d}_{A_{k}, B_{h}}\right) \underline{N}\left(A_{k}\right) \underline{N}\left(B_{h}\right)
$$

If we select the tessellations $\underline{\mathcal{A}}^{n}, \underline{\mathcal{B}}^{n}, \overline{\mathcal{A}}^{n}, \overline{\mathcal{B}}^{n}$, in such a way that their elements have area $(16+\delta) \gamma(n)$, combining (11) and (12) bounds, according to Lemma 1 , it results:

$$
\begin{array}{r}
\frac{(16+\delta)^{2} n^{2}}{4} \sum_{A_{k} \in \underline{A}^{n}} \sum_{B_{h} \in \underline{B}^{n}} \mu^{n}\left(\bar{d}_{A_{k}, B_{h}}\right) \gamma^{2}(n) \leq \mu_{\mathcal{L}}^{n} \leq \\
(16+\delta)^{2} 4 n^{2} \sum_{A_{k} \in \overline{\mathcal{A}}^{n}} \sum_{B_{h} \in \overline{\mathcal{B}}^{n}} \mu^{n}\left(\underline{d}_{A_{k}, B_{h}}\right) \gamma^{2}(n)
\end{array}
$$

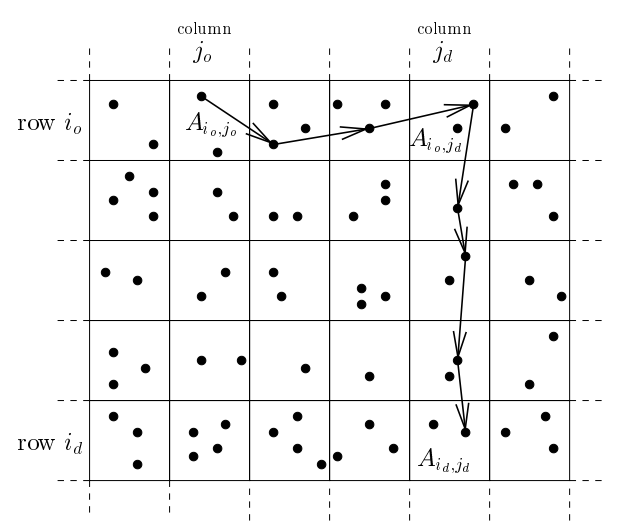

Figure 5: Possible logical route from a source located in squarelet $A_{i_{o}, j_{o}}$ to a destination located in squarelet $B_{i_{d}, j_{d}}$. Dots denote home-points and arrows represent logical hops of the path: the actual forwarding occurs when nodes corresponding to the selected home-points get in contact with each other.

Note that $\bar{d}_{A_{k}^{n}, B_{h}^{n}}-\underline{d}_{A_{k}^{n}, B_{h}^{n}} \leq 2 \sqrt{2(16+\delta) \gamma(n)}$. Then under the condition $f(n) \sqrt{\gamma(n)}=o(1)$, it results:

$$
\begin{aligned}
& \sum_{A_{k} \in \overline{\mathcal{A}}^{n}} \sum_{B_{h} \in \overline{\mathcal{B}}^{n}} \eta\left(f(n) \underline{d}_{A_{k}, B_{h}}\right) \gamma^{2}(n) \sim \\
\sim & \sum_{A_{k} \in \underline{A}^{n}} \sum_{B_{h} \in \underline{B}^{n}} \eta\left(f(n) \bar{d}_{A_{k}, B_{h}}\right) \gamma^{2}(n) \sim \\
\sim & \int_{X \in I_{\mathcal{L}}} \int_{Y \in E_{\mathcal{L}}} \eta(f(n)\|X-Y\|) d X d Y
\end{aligned}
$$

being $\mu(d)$ non increasing and summable. Indeed, observe that, i) (13) and (14) can be interpreted, respectively, as lower Riemann sum and upper Riemann sum of $\int_{X \in I_{\mathcal{L}}} \int_{Y \in E_{\mathcal{L}}} \eta(f(n) \| X-$ $Y \|) d X d Y$; ii) since $f(n) \sqrt{\gamma(n)}=o(1)$, the mesh size of the partitions associated to Riemann sums vanishes to 0 as $n \rightarrow \infty$.

According to Proposition 1, the evaluation of $\mu_{\mathcal{L}}^{n}$ permits to obtain an upper bound to the transport capacity of the GRGG. Considering, indeed, any simple, regular, closed curve $\mathcal{L}$, under any uniform permutation traffic, the number of flows crossing $\mathcal{L}$ (i.e. connections whose source lies in $I_{\mathcal{L}}$ and destination in $E_{\mathcal{L}}$ ) w.h.p. is $\Theta(n)$, thus by (10) it results:

$$
\lambda \leq \Theta\left(n g(n) \int_{X \in I_{\mathcal{L}}} \int_{Y \in E_{\mathcal{L}}} \eta(f(n)\|X-Y\|) d X d Y\right)
$$

To evaluate the order of magnitude of $\lambda$, the following result comes in handy:

Proposition 2. Under the assumption $\int x^{3} \eta(x) d x<\infty$, for any convex, simple, regular, closed curve $\mathcal{L}$ :

$$
f^{2}(n) \int_{X \in I_{\mathcal{L}}} \int_{Y \in E_{\mathcal{L}}} \eta(f(n)\|X-Y\|) d X d Y=\Theta\left(\frac{1}{f(n)}\right)
$$

from which we obtain $\lambda=\Theta\left(\frac{1}{f(n)}\right)$. The proof is reported in Appendix C.

\subsection{Optimal Routing Scheme}

Here we show that the above upper bound on network capacity is tight presenting a simple routing scheme that achieves per-node throughput $\Theta\left(\frac{1}{f(n)}\right)$. 
We partition the network area $\mathcal{O}$ into a regular square tessellation $\mathcal{A}^{n}$ whose elements have area equal to $c^{2} / f^{2}(n)$, being $c$ an appropriate constant. Squarelets $A_{i, j}^{n}$ are indexed by tuple $(i, j)$, where $i$ is the row index, and $j$ is the column index. We adopt the following routing scheme, illustrated in Fig. 5: traffic originated by a node in $A_{i_{o}, j_{o}}^{n}$ and destined to a node in $A_{i_{d}, j_{d}}^{n}$ is first routed along a (logical) horizontal path through a sequence of randomly selected relay home-points residing in contiguous squarelets up to reach a node whose home-point is in $A_{i_{o}, j_{d}}^{n}$, and then routed through a similar (logical) vertical path up to its final destination.

An upper bound to the total traffic flowing between two adjacent squarelets $A$ and $B$ can be easily obtained as follows. The traffic between two adjacent horizontal squarelets cannot exceed the total traffic produced by all sources lying in the same row. Similarly, the traffic between two adjacent vertical squarelets cannot exceed the total traffic sent to destinations lying in the same column. Since $\gamma(n)=o\left(1 / f^{2}(n)\right)$, thanks to Lemma 1 we can upper bound the traffic flowing between any two adjacent squarelets by $\lambda \frac{2 c n}{f(n)}$.

On the other hand, considering the GRGG associated to the network, the number of edges connecting vertices (home-points) in $A$ to vertices (home-points) in $B$ is equal to $N(A) N(B)$. It follows that an upper bound to the traffic traversing each individual edge connecting squarelets $A$ and $B$ is given by:

$$
\frac{\lambda \frac{2 c n}{f(n)}}{\underline{N}(A) \underline{N}(B)}
$$

where w.h.p. uniformly over the tessellation, $\underline{N}(A)=\underline{N}(B)=$ $\frac{c^{2} n}{2 f^{2}(n)}$, since $\gamma(n)=o\left(1 / f^{2}(n)\right)$. Traffic can be sustained if no edge is overloaded; this is true w.h.p. if:

$$
\frac{\lambda \frac{2 c n}{f(n)}}{\underline{N}(A) \underline{N}(B)}=\lambda \frac{8 f^{3}(n)}{c^{3} n} \leq \mu^{n}\left(\bar{d}_{A, B}\right)=g(n) \eta(\sqrt{5} c)
$$

being $\bar{d}_{A, B}=\frac{\sqrt{5} c}{f(n)}$. Note that $c$ must be chosen is such a way that $\eta(\sqrt{5} c)>0$. As a result, the maximum sustainable per-node traffic with the proposed routing scheme is:

$$
\lambda=\Theta\left(\frac{g(n) \eta(\sqrt{5} c)}{\frac{8 f^{3}(n)}{c^{3} n}}\right)=\Theta\left(\frac{1}{f(n)}\right)
$$

\section{ANALYSIS IN SUB-CRITICAL REGIME}

In this section we briefly discuss what happens when the condition $f(n) \sqrt{\gamma(n)}=o(1)$ is violated. More specifically, we limit our discussion to the strictly sub-critical regime (i.e., when $f(n) \sqrt{\gamma(n)}=\omega(1))$ leaving for future investigations the critical case $f(n) \sqrt{\gamma(n)}=\Theta(1)$.

Unfortunately, the insensitiveness of network capacity to the shape of $s(d)$ is lost in sub-critical regime. Here we focus on the worst case among all possible shapes $s(d)$, which provides a lower bound to network capacity in sub-critical regime. First we introduce the following result.

Proposition 3. If $f(n) \sqrt{\gamma(n)}=\omega(1)$, values of $R_{T}(n)<$ $\sqrt{\gamma(n)}$ may fail even to guarantee network connectivity.

PROOF. we restrict our investigation to the case in which $s(d)$ has finite support, i.e., $s(d)=0$ for $d>d_{0} \in \mathbb{R}^{+}$; in this case, considering two nodes $i$ and $j$ and applying the triangular inequality to the distance between them we obtain:

$$
\left\|X_{i}(t)-X_{j}(t)\right\| \geq\left\|X_{i}^{h}-X_{j}^{h}\right\|-2 \frac{d_{0}}{f(n)}
$$

which implies that, whenever $\left\|X_{i}^{h}-X_{j}^{h}\right\|=\omega\left(\frac{1}{f(n)}\right)$, then $\left\|X_{i}(t)-X_{j}(t)\right\| \sim\left\|X_{i}^{h}-X_{j}^{h}\right\|=\omega\left(\frac{1}{f(n)}\right)$, i.e., for sufficiently large $n$, the transmission capacity $\mu_{i j}^{n}$ between node pairs $(i, j)$ such that $\left\|X_{i}^{h}-X_{j}^{h}\right\|=\omega\left(\frac{1}{f(n)}\right)$ is null unless $R_{T}(n)=\Theta\left(\left\|X_{i}^{h}-X_{j}^{h}\right\|\right)$.

On the other hand, it was shown in [1] for the Uniform model that the probability that every node $i$ in the network finds at least another node $j$ whose home-point $X_{j}^{h}$ has distance from $X_{i}^{h}$ less than $k(n)$, asymptotically satisfies:

$$
\operatorname{Pr}\left\{\forall i, \exists j \neq i:\left\|X_{i}^{h}-X_{j}^{h}\right\|<k(n)\right\}<\beta<1
$$

for any $k(n) \leq \sqrt{\frac{\log (n)}{n}}$. As a consequence the network may result asymptotically disconnected with strictly positive probability as long as $R_{T}(n) \leq \sqrt{\frac{\log (n)}{n}}$.

The previous result can be easily extended to the clustered model, since the $m(n)$ cluster centers are uniformly and independently located within the area. It turns out that the probability that every node $i$ in the network finds another node $j$ belonging to a different cluster, such that the distance between the corresponding home points is less than $k(n)$, is asymptotically strictly less than 1 when $k(n)<\sqrt{\left.\frac{\log (m(n))}{m(n)}\right)}$ :

$\operatorname{Pr}\left\{\forall\right.$ cluster $\left.c, \forall i \in c, \exists j \notin c:\left\|X_{i}^{h}-X_{j}^{h}\right\|<k(n)\right\}<\beta<1$

for any $k(n)<\sqrt{\frac{\log (m(n))}{m(n)}}$. Thus also in this case the network is disconnected with strictly positive probability as long as $R_{T}(n)=$ $o\left(\sqrt{\frac{\log (n)}{n}}\right)$.

As a consequence, node mobility plays no significant role, and the system behaves as if nodes were fixed. Therefore, the GuptaKumar result (whose extension to the clustered model is rather straightforward) can be applied in sub-critical regime to estimate the network capacity when $s\left(d_{0}\right)=0$, for some $d_{0}$. The resulting per node capacity is:

$$
\Theta\left(\frac{1}{n R_{T}(n)}\right)=\Theta\left(\sqrt{\frac{m(n)}{n^{2} \log (m(n))}}\right)
$$

In the case $s(d)>0$ for any $d$, then all pairs of nodes occasionally meet, thus network connectivity is guaranteed also by using transmission ranges $R_{T}(n)=o(\sqrt{\gamma(n)})$. As a consequence, the system capacity can be in general increased with respect to the case in which $s(d)$ has finite support.

\section{CONCLUSIONS}

In this paper, we have extended the analysis of the capacity scaling properties in mobile ad-hoc networks by considering heterogeneous nodes and spatial inhomogeneities, two common features widely recognized in realistic mobility traces. We have shown how to map the problem onto a Maximum Concurrent Flow (MCF) problem over an associated Generalized Random Geometric Graph (GRGG). Our methodology allows to identify the scaling laws of a general class of mobile wireless networks, and to precisely determine under which conditions the mobility of nodes can indeed be exploited to increase the per-node throughput.

\section{REFERENCES}

[1] P. Gupta, P.R. Kumar, "The capacity of wireless networks", IEEE Trans. on Information Theory, vol. 46, n.2, pp. 388-404, Mar. 2000 
[2] M. Grossglauser, D.N.C. Tse, "Mobility increases the capacity of ad hoc wireless networks", IEEE Trans. on Networking, vol. 10, n.2, pp. 477-486, Aug. 2002

[3] Delay Tolerant Network Research Group: www.dtnrg.org

[4] A. Chaintreau, P. Hui, J. Crowcroft, C. Diot, R. Gass, J. Scott, "Impact of Human Mobility on the Design of Opportunistic Forwarding Algorithms", in Proc. IEEE INFOCOM '06, Barcelona, Spain, April 2006.

[5] J. Burgess, B. Gallagher, D. Jensen, B. N. Levine, "MaxProp: Routing for Vehicle-Based Disruption-Tolerant Networking", in Proc. IEEE INFOCOM 2006, Barcelona, Spain, April 2006.

[6] N. Sarafijanovic-Djukic, M. Piorkowski, and M. Grossglauser, "Island Hopping: Efficient Mobility-Assisted Forwarding in Partitioned Networks", IEEE SECON 2006, Reston, VA, Sep. 2006.

[7] P. Juang, H. Oki, Y. Wang, M. Martonosi, L.-S. Peh, D. Rubenstein, "Energy-Efficient Computing for Wildlife Tracking: Design Tradeoffs and Early Experiences with ZebraNet", in Proc. ASPLOS-X, Oct. 2002, San Jose, CA.

[8] W. Zhao, M. Ammar, and E. Zegura, "A message ferrying approach for data delivery in sparse mobile ad hoc networks", In Proc. MobiHocŠ 04, 2004.

[9] A. Pentland, R. Fletcher, A. Hasson, "Daknet: rethinking connectivity in developing nations",IEEE Computer, 37:78-83, 2004.

[10] S. Toumpis and A. Goldsmith, "Large wireless networks under fading, mobility, and delay constraints", IEEE INFOCOM, Hong Kong, China, Mar. 2004

[11] A.El Gamal, J. Mammen, B. Prabhakar, and D. Shah, "Throughput-Delay Trade-off in Wireless Networks", IEEE INFOCOM, Hong Kong, China, Mar. 2004

[12] G. Sharma, R. R. Mazumdar and N. B. Shroff, "Delay and Capacity Trade-offs in Mobile Ad Hoc Networks: A Global Perspective" IEEE INFOCOM, Barcelona, Spain, Apr. 2006

[13] W.-J. Hsu and A. Helmy, "On Nodal Encounter Patterns in Wireless LAN Traces", in Proc. WiNMee '06, Boston, MA, 2006.

[14] J. Leguay, T. Friedman, V. Conan, "Evaluating Mobility Pattern Space Routing for DTNs", in Proc. IEEE INFOCOM '06, Barcelona, Spain, April 2006.

[15] M. Balazinska, P. Castro, "Characterizing Mobility and Network Usage in a Corporate Wireless Local-Area Network", in Proc. ACM MobiSys '03, San Francisco, CA, May 2003.

[16] J. H. Kang, W. Welbourne, B. Stewart, G. Borriello, "Extracting Places from Traces of Locations", ACM Mobile Computing and Communications Review, 9(3), July 2005.

[17] M. Kim, D. Kotz, S. Kim, "Extracting a mobility model from real user traces", in Proc. IEEE INFOCOM '06, Barcelona, Spain, April 2006.

[18] S.N. Diggavi, M. Grossglauser, D.N.C. Tse, "Even one-dimensional mobility increases ad hoc wireless capacity", IEEE Trans. on Information Theory, vol. 51, n. 11, pp. 3947-3954, Nov. 2005

[19] R. M. Moraes, H. R. Sadjadpour and J. J. Garcia-Luna Aceves, "Mobility-Capacity-Delay Trade-off in Wireless Ad Hoc Networks," Elsevier Journal on ad hoc networks, July 2005

[20] M. Garetto, P. Giaccone, E. Leonardi, "On the Capacity of Ad Hoc Wireless Networks Under General Node Mobility”, IEEE Infocom 2007, Anchorage, AK, May 2007

[21] S. R. Kulkarni, P. Viswanath, "A Deterministic Approach to Throughput Scaling in Wireless Networks," IEEE Trans. on Infornation Theory, vol. 50, no. 6 , pp. 1041-1049, June 2004

[22] S. Toumpis, "Capacity bounds for three classes of wireless networks: asymmetric, cluster, and hybrid", ACM MobiHoc, pp. 133-144, Tokyo, Japan, May 2004

[23] C. Peraki, S. D. Servetto "On the Maximum Stable Throughput Problem in Random Networks with Directional Antennas," ACM MobiHoc, Annapolis, MD (USA), 2003

[24] U.C. Kozat and L. Tassiulas, "Throughput capacity of random ad hoc networks with infrastructure support" ACM MobiCom, pp. 55-65, San Diego, CA, Sep. 2003

[25] A. Agarwal and P. R. Kumar, "Capacity bounds for ad-hoc and hybrid wireless networks", ACM Computer Communications Review, vol. 34, no. 3, pp. 71-81, July 2004

[26] B. Liu, Z. Liu, and D. Towsley, "On the capacity of hybrid wireless networks", IEEE INFOCOM, vol. 2, pp. 1543-1552, San Francisco, CA, Apr. 2003

[27] M. Penrose, Random Geometric Graphs, Oxford University Press, 2003.

[28] Y. Aumann and Y. Rabani, "An $O(\log k)$ Approximate Min-Cut Max-Flow Theorem and Approximation Algorithm", SIAM Journal on Computing, vol. 27, n. 1, 1998

[29] R. Motwani, P. Raghavan, Randomized algorithms, Cambridge University Press, 1995

\section{APPENDIX}

\section{A. PROOF OF LEMMA 1}

First we start with the Uniform model. Given an element $A_{k}^{n}$ of tessellation $\mathcal{A}^{n}$, by definition

$$
N\left(A_{k}^{n}\right)=\sum_{i=1}^{n} \mathbb{I}_{X_{i}^{h} \in A_{k}^{n}}
$$

with $\mathbb{I}_{X_{i}^{h} \in A_{k}^{n}}$ i.i.d. Bernoullian random variables with mean $p_{1}=$ $E\left[\mathbb{I}_{X_{i}^{h} \in A_{k}^{n}}\right]^{k}=\left|A^{n}\right|$. By applying Chernoff bounds [29] we get:

$$
\begin{gathered}
\operatorname{Pr}\left\{N\left(A_{k}^{n}\right)<\frac{1}{2} E\left[N\left(A_{k}^{n}\right)\right]=\frac{1}{2} n p_{1}\right\}<e^{-n p_{1} / 8} \\
\operatorname{Pr}\left\{N\left(A_{k}^{n}\right)>2 E\left[N\left(A_{k}^{n}\right)\right]\right\}<(e / 4)^{n p_{1}}<e^{-n p_{1} / 8}
\end{gathered}
$$

Thus, setting: $\left|A^{n}\right|=16 \frac{\log (n)}{n}$, we obtain:

$$
\begin{aligned}
\operatorname{Pr}\left\{\frac{1}{2} E\left[N\left(A_{k}^{n}\right)\right]\right. & \left.<N\left(A_{k}^{n}\right)<2 E\left[N\left(A_{k}^{n}\right)\right]\right\} \geq \\
& \geq 1-2 e^{-2 \log n}=1-2 n^{-2}
\end{aligned}
$$

At last by sub-additivity of probability measures:

$$
\begin{gathered}
\operatorname{Pr}\left\{\cap_{k}\left\{\frac{1}{2} E\left[N\left(A_{k}^{n}\right)\right]<N\left(A_{k}^{n}\right)<2 E\left[N\left(A_{k}^{n}\right)\right]\right\}\right\} \geq \\
1-\sum_{k} \operatorname{Pr}\left\{N\left(A_{k}^{n}\right)<\frac{1}{2} E\left[N\left(A_{k}^{n}\right)\right] \text { or } N\left(A_{k}^{n}\right)>2 E\left[N\left(A_{k}^{n}\right)\right]\right\} \geq \\
\geq 1-n 2 n^{-2}=1-\frac{2}{n}
\end{gathered}
$$

In the case of clustered model with $m(n)=n^{\nu}, \nu \in[0,1)$, and $\lim _{n \rightarrow \infty} m(n) r^{2}(n)=0$, consider a sequence of regular tessellations $\mathcal{A}_{n}$, such that $\left|A^{n}\right|>16 \gamma(n)$. Since centers of clusters are distributed according to a uniform distribution, the number of clusters $N_{c}\left(A_{k}^{n}\right)$ whose center fall within $A_{k}^{n}$, satisfies, uniformly over the tessellation, w.h.p :

$$
1 / 2 E\left[N_{c}\left(A_{k}^{n}\right)\right]<N_{c}\left(A_{k}^{n}\right)<2 E\left[N_{c}\left(A_{k}^{n}\right)\right]
$$

Let $n_{c}$ be the number of nodes belonging to cluster $c$, $1<c<m(n)$; for any $\epsilon>0$ with high probability, $\frac{(1-\epsilon)}{m(n)}<n_{c}<\frac{(1+\epsilon)}{m(n)}$, uniformly over $c$. Indeed, considering a particular cluster $c$, by definition it results: $n_{c}=\sum_{1}^{n} \mathbb{I}_{i \in c}$, being $\mathbb{I}_{i \in c}$ i.i.d. Bernoullian random variables with average $p_{1}=1 / m(n)$; thus applying again the Chernoff bound

$$
\begin{aligned}
& \operatorname{Pr}\left\{\frac{(1-\epsilon) n}{m(n)}<n_{c}<\frac{(1+\epsilon) n}{m(n)}\right\} \geq \\
& \quad \geq 1-2 \exp \left(-\frac{\left(\epsilon \frac{n}{m(n)}\right)^{2}}{2 \frac{n}{m(n)}}\right)=1-2 \exp \left(-\frac{\epsilon n^{1-\nu}}{2}\right)
\end{aligned}
$$

As a consequence:

$$
\begin{gathered}
\operatorname{Pr}\left\{\cap_{c}\left\{\frac{(1-\epsilon) n}{m(n)}<n_{c}<\frac{(1+\epsilon) n}{m(n)}\right\}\right\} \geq 1- \\
\sum_{c} \operatorname{Pr}\left\{n_{c} \notin\left[\frac{(1-\epsilon) n}{m(n)}, \frac{(1+\epsilon) n}{m(n)}\right]\right\} \geq 1-2 n \exp \left(-\frac{\epsilon n^{1-\nu}}{2}\right)
\end{gathered}
$$

which tends to 0 when $n \rightarrow \infty$. Moreover, for any element $A_{k}^{n}$, under the condition $\left|A^{n}\right| \geq(16+\delta) \gamma(n)$, for any small $\delta>0$, defining with $\underline{A}_{k}^{n}$ the subset of its points whose distance from its frontier is greater than $r(n)$, and with $\bar{A}_{k}^{n}$ the superset of $A_{k}^{n}$ comprising points in $A_{k}^{n}$ plus those points whose distance from points of $A_{k}^{n}$ is not greater than $r(n)$, it is of immediate verification that:

$$
\sum_{c} \sum_{i} \mathbb{I}_{i \in c} \mathbb{I}_{c \in \underline{A}_{k}^{n}} \leq N\left(A_{k}^{n}\right) \leq \sum_{c} \sum_{i} \mathbb{I}_{i \in c} \mathbb{I}_{c \in \bar{A}_{k}^{n}}
$$


Since $\left|\underline{A}_{k}^{n}\right| \sim\left|\bar{A}_{k}^{n}\right| \sim\left|A_{k}^{n}\right|$, it follows that $\left|\underline{A}_{k}^{n}\right|>16 \frac{\log (m(n))}{m(n)}$, for sufficiently large $n$; thus previously obtained results can be applied to bound $\sum_{c} \mathbb{I}_{c \in \underline{A}_{k}^{n}}, \sum_{i} \mathbb{I}_{i \in c}$, and $\sum_{c} \mathbb{I}_{c \in \bar{A}_{k}^{n}}$, and the assert follows, letting $\epsilon \rightarrow 0$.

\section{B. PROOF OF THEOREM 2}

By construction:

$$
\begin{aligned}
& \operatorname{Pr}\left\{d_{i j}^{(n)} \leq \frac{c_{T}}{\sqrt{n}}\right\}=\int_{X_{i} \in \mathcal{O}} \int_{X_{j} \in B\left(X_{i}, c_{T} / \sqrt{n}\right)} \phi^{n}\left(X_{j}-X_{j}^{h}\right) \\
& \phi^{n}\left(X_{i}-X_{i}^{h}\right) d X_{j} d X_{i}
\end{aligned}
$$

while $\mu_{i j}^{* n}$ is given by (5); thus, to prove the assert it is sufficient to show that w.h.p., for some $\epsilon>0$, $\lim _{n \rightarrow \infty} \prod_{k \neq i, j} \int_{X_{k} \in A_{\Delta}\left(X_{i}, X_{j}\right)} \phi^{n}\left(X_{k}-X_{k}^{h}\right) d X_{k}=$ $\prod_{k \neq i, j} E\left[\mathbb{I}_{X_{k}^{(n)}(t) \in A_{\Delta}\left(X_{i}, X_{j}\right)} \mid \mathcal{F}_{n}\right]>\epsilon$ for every $X_{i} \in \mathcal{O}$ and $X_{j} \in B\left(X_{i}, c_{T} / \sqrt{n}\right)$. Note that, by triangular inequality, $\lim _{n \rightarrow \infty} \int_{X_{k} \notin A_{\Delta}\left(X_{i}, X_{j}\right)} \phi^{n}\left(X_{k}-X_{k}^{h}\right) d X_{k} \leq$

$\leq \int_{X_{k} \in B\left(X_{i}, 2 \frac{c_{T}}{\sqrt{n}}\right)} \phi^{n}\left(X_{k}-X_{k}^{h}\right) d X_{k} \rightarrow 0$ since, by hypothesis, $\frac{f(n)}{\sqrt{n}}=o(1)$. Thus for any $X_{i}$ and $X_{j} \in B\left(X_{i}, c_{T} / \sqrt{n}\right)$, defined for short: $p_{k}^{n}=E\left[\mathbb{I}_{X_{k}^{(n)} \notin A_{\Delta}\left(X_{i}, X_{j}\right)} \mid \mathcal{F}_{n}\right]$, it results:

$$
\lim _{n \rightarrow \infty} p_{k}^{n}=0 \quad \forall k
$$

In addition, thanks to (3), with high probability, uniformly over $X_{i}$ and $X_{j} \in B\left(X_{i}, c_{T} / \sqrt{n}\right)$ :

$$
\lim _{n \rightarrow \infty} \sum_{k \neq i, j}^{n} p_{k}^{n}<2 c_{T} M
$$

To conclude the proof, observe that $\lim _{n \rightarrow \infty} \prod_{k \neq i, j}\left(1-p_{k}^{n}\right)>$ $\epsilon>0$ by the continuity of $\log$ function is equivalent to: $\lim _{n \rightarrow \infty} \sum_{k \neq i, j} \log \left(1-p_{k}^{n}\right)>\log \epsilon>-\infty$ and that $\log (1-x)>-2 x$ for any $0 \leq x \leq x_{0}$, with $x_{0} \approx 0.8$ solution of equation $1-x_{0}=\exp \left(-2 x_{0}\right)$. At last, since for $n$ sufficiently large thanks to (17) and (18) we can assume all $p_{k}^{n}<x_{0}$, we get $\lim _{n \rightarrow \infty} \sum_{k \neq i, j} \log \left(1-p_{k}^{n}\right) \geq-\sum_{k \neq i, j} 2 p_{k}^{n}>-4 c_{T} M$.

\section{PROOF OF PROPOSITION 2}

Fist we consider the case $f(n)=\Theta(1)$; without lack of generality we can assume $f(n)=1$. In the particular case $\eta(d)=1$ (which is essentially the Grossglauser-Tse case) the result is immediate, since, for any $\mathcal{L}$ :

$$
\int_{I_{\mathcal{L}}} \int_{E_{\mathcal{L}}} \eta(\|X-Y\|) d X d Y=\int_{I_{\mathcal{L}}} d X \int_{E_{\mathcal{L}}} d Y=\Theta(1)
$$

In the more general case let $D=\sup _{d} \eta(d)>\frac{\eta(0)}{2}$, and for any point $X \in I_{\mathcal{L}}$ let $X_{\mathcal{L}}$ its projection on curve $\mathcal{L}$. At last, let $I_{\mathcal{L}}^{D / 2}$ the set of points in $I_{\mathcal{L}}$ whose distance from $\mathcal{L}$ is less or equal than $D / 2$.

Note that $\eta(d) \geq \eta(0) / 2 \mathbb{I}_{d<D}$ and remind that by triangular inequality $\left\|X-X_{\mathcal{L}}\right\|+\left\|Y-X_{\mathcal{L}}\right\| \geq\|X-Y\|$, thus:

$$
\begin{gathered}
\int_{X \in I_{\mathcal{L}}} \int_{Y \in E_{\mathcal{L}}} \eta(\|X-Y\|) d X d Y \geq \\
\geq \int_{X \in I_{\mathcal{L}}} \int_{Y \in E_{\mathcal{L}}} \eta(\|X-Y\|) \mathbb{I}_{\|X-Y\| \leq D} d X d Y \geq \\
\geq \int_{X \in I_{\mathcal{L}}} \int_{Y \in E_{\mathcal{L}}} \frac{\eta(0)}{2} \mathbb{I}_{\left\|X-X_{\mathcal{L}}\right\| \leq D / 2} \mathbb{I}_{\left\|Y-X_{\mathcal{L}}\right\| \leq D / 2} d X d Y= \\
=\int_{X \in I_{\mathcal{L}}^{D / 2}} \frac{\eta(0)}{2}\left[\int_{E_{\mathcal{L}}} \mathbb{I}_{\left\|Y-X_{\mathcal{L}}\right\| \leq D / 2} d Y\right] d X \geq \frac{\eta(0)}{2}\left|I_{\mathcal{L}}^{D / 2}\right| \pi \frac{D^{2}}{4}
\end{gathered}
$$

where the last inequality holds since the set of points in $E_{\mathcal{L}}$ whose distance from $X_{\mathcal{L}}$ is not greater than $D / 2$ covers a semicircle of radius $D / 2$, being $\mathcal{L}$ convex.

On the other side it is immediate to verify:

$$
\int_{I_{\mathcal{L}}} \int_{E_{\mathcal{L}}} \eta(\|X-Y\|) d X d Y \leq \eta(0)\left|I_{\mathcal{L}} \| E_{\mathcal{L}}\right|
$$

and thus since both $D$ and $\left|I_{\mathcal{L}}^{D / 2}\right| \approx|\mathcal{L}| D / 2$ are finite, we get the assert.

In case $f(n)=\omega(1)$, to obtain a lower bound it is sufficient to define $D^{n}=\sup _{d} \eta(f(n) d)>\frac{\eta(0)}{2}$, (note that $D^{n}$ scales as $1 / f(n))$ and repeat the previous arguments. To obtain an upper bound, first suppose the support of $\eta(d)$ is finite, i.e., there exists $d_{0}$ such that $\eta(d)=0$ for $d>d_{0}$. In such a case $\eta(d) \leq \eta(0) \mathbb{I}_{d<d_{0}}$. Define $I_{\mathcal{L}}^{\frac{d_{0}}{f(n)}}$ the set of points in $I_{\mathcal{L}}$ whose distance from $\mathcal{L}$ is less or equal than $d_{0} / f(n)$. It results:

$$
\begin{gathered}
\int_{X \in I_{\mathcal{L}}} \int_{Y \in E_{\mathcal{L}}} \eta(f(n)\|X-Y\|) d X d Y \leq \\
\leq \int_{X \in I_{\mathcal{L}}} \int_{Y \in E_{\mathcal{L}}} \eta(0) \mathbb{I}_{\|X-Y\| \leq \frac{d_{0}}{f(n)}} d X d Y \leq \\
\leq \int_{X \in I_{\mathcal{L}}} \int_{Y \in E_{\mathcal{L}}} \eta(0) \mathbb{I}_{\left\|X-X_{\mathcal{L}}\right\| \leq \frac{d_{0}}{f(n)}} \mathbb{I}_{\left\|Y-X_{\mathcal{L}}\right\| \leq \frac{d_{0}}{f(n)}} d X d Y= \\
=\int_{X \in I_{\mathcal{L}}^{\frac{d_{0}}{f(n)}}} \eta(0)\left[\int_{E_{\mathcal{L}}} \mathbb{I}_{\left\|Y-X_{\mathcal{L}}\right\| \leq \frac{d_{0}}{f(n)}} d Y\right] d X \leq \eta(0)\left|I_{\mathcal{L}}^{\frac{d_{0}}{f(n)}}\right| 2 \pi \frac{d_{0}^{2}}{4 f^{2}(n)} \\
\text { being }\left|I_{\mathcal{L}}^{\frac{d_{0}}{f(n)}}\right| \approx \mid \mathcal{L} \| \frac{d_{0}}{f(n)} .
\end{gathered}
$$

Consider now the case in which the support of $\eta(d)$ is unlimited; by assumption $\sum_{i}(i+1)^{3} \eta(i)<\infty$. To get a new upper bound note that:

$$
\eta(d) \leq \sum_{i} \eta(i) \mathbb{I}_{i \leq d \leq(i+1)} \leq \sum_{i} \eta(i) \mathbb{I}_{d \leq(i+1)}
$$

now:

$$
\begin{aligned}
& \int_{I_{\mathcal{L}}} \int_{E_{\mathcal{L}}} \eta(f(n)\|X-Y\|) d X d Y \leq \\
& \leq \int_{I_{\mathcal{L}}} \int_{E_{\mathcal{L}}} \sum_{i} \eta(i) \mathbb{I}_{f(n)\|X-Y\| \leq i+1} d X d Y \leq \\
& \quad \leq \sum_{i} \eta(i) \int_{I_{\mathcal{L}}} \int_{E_{\mathcal{L}}} \mathbb{I}_{f(n)\|X-Y\|<i+1} d X d Y
\end{aligned}
$$

where the last equality is made possible by the monotone convergence theorem. Bounding each term as before we get the assert. 